\title{
CRECIMIENTO DE PLANTAS Y RENDIMIENTO DE TOMATE EN DIVERSAS RELACIONES NITRATO/AMONIO Y CONCENTRACIONES DE BICARBONATO
}

\author{
PLANT GROWTH AND TOMATO YIELD AT SEVERAL NITRATE/AMMONIUM RATIOS AND \\ BICARBONATE CONCENTRATIONS
}

\author{
Saúl Parra Terraza ${ }^{*}$ Praxédes Lara Murrieta, Manuel Villarreal Romero y Sergio Hernández Verdugo
}

Facultad de Agronomía, Universidad Autónoma de Sinaloa. Carretera Culiacán-El dorado Km 17.5. Apartado Postal 726. 80000, Culiacán, Sinaloa. Tel. y Fax 01 (667) 846-10-84, Ext. 107

*Autor para correspondencia (psaul@uas.uasnet.mx)

\section{RESUMEN}

Aunque se ha reportado el efecto principal de diferentes relaciones de nitrato/amonio y de concentraciones de bicarbonato en la solución nutritiva sobre el crecimiento de las plantas, se conoce poco de la influencia de la interacción de ambos factores sobre el tomate (Lycopersicon esculentum Mill.). Por ello se hicieron dos estudios para determinar la influencia de tres relaciones porcentuales nitrato/amonio (100/0, 85/15 y 70/30) y tres concentraciones de $\mathrm{HCO}_{3}^{-}$ $\left(0,2.5\right.$ y 5 mol $\left._{c} \mathrm{~m}^{-3}\right)$ en la solución nutritiva, sobre el crecimiento, rendimiento y composición mineral de tomate cv. 'Slolly F-1' desarrollado en hidroponía. La relación 70/30 de nitrato/amonio redujo $(P \leq 0.05)$ el peso seco de raíz y el volumen radical, mientras que la concentración de $5 \mathbf{m o l}_{\mathrm{c}} \mathrm{HCO}_{3}{ }^{-} \mathbf{m}^{-3}$ disminuyó el diámetro de tallo, el peso seco de hojas, el volumen radical y la relación vástago/raíz de las plantas jóvenes. La concentración de calcio en hojas y tallos de plantas jóvenes y plantas adultas se redujo $(\mathrm{P} \leq 0.05)$ con la relación 70/30; en contraste, la concentración de fósforo en dichos órganos se incrementó $(\mathrm{P} \leq 0.05)$. La adición de $5 \mathrm{~mol}_{\mathrm{c}} \mathrm{HCO}_{3}{ }^{-} \mathrm{m}^{-3}$ a la solución nutritiva redujo $(\mathrm{P} \leq \mathbf{0 . 0 5})$ la concentración de fósforo en hojas y tallos de plantas jóvenes, y en tallos y frutos de plantas adultas.

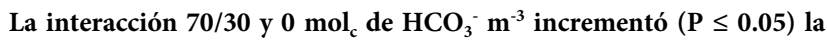
altura de plantas jóvenes y redujo la concentración de magnesio en hojas y tallos. Sustituir $30 \%$ del nitrógeno nítrico con nitrógeno amoniacal o añadir $5 \mathrm{~mol} \mathrm{HCO}_{3}^{-} \mathrm{m}^{-3}$ a la solución nutritiva en la etapa de planta joven, redujo algunos indicadores del crecimiento pero no afectó al rendimiento de tomate.

Palabras clave: Lycopersicon esculentum, crecimiento, relación nitrato/amonio, bicarbonato, hidroponía.

\section{SUMMARY}

The main effect of different nitrate/ammonium ratios and bicarbonate concentrations in the nutrient solution on growth of plants has been reported, but little is known of the influence of the interaction of these two factors on tomato (Lycopersicon esculentum Mill.). Therefore, two studies were conducted to determine the influence of three nitrate/ammonium percentage ratios $(100 / 0,85 / 15$ and 70/30) and three concentrations of $\mathrm{HCO}_{3}^{-}\left(0,2.5\right.$, and $\left.5 \mathrm{~mol}_{\mathrm{c}} \mathrm{m}^{-3}\right)$ in the nutrient solution on growth, yield and mineral composition of tomato 'cv. Slolly F-1' grown in hydroponics. The 70/30 nitrate/ammonium ratio reduced $(\mathrm{P} \leq 0.05)$ dry weight and the root volume, while the concentration of $5 \mathrm{~mol}_{\mathrm{c}} \mathrm{HCO}_{3}^{-} \mathrm{m}^{-3}$ decreased diameter of stem, leaf dry weight, root volume and stem/root ratio of young plants. The calcium concentration in leaves and stems of young plants and mature plants decreased $(P \leq 0.05)$ with the $70 / 30$ ratio. In contrast, the phosphorus concentration in such organs increased $(\mathrm{P}$ $\leq 0.05)$. The addition of $5 \mathrm{~mol}_{\mathrm{c}} \mathrm{HCO}_{3}{ }^{-} \mathrm{m}^{-3}$ to the nutrient solution reduced $(\mathrm{P} \leq 0.05)$ the phosphorus concentration in leaves and stems of young plants, and stems and fruits of mature plants. The 70/30 and 0 mol $_{\mathrm{c}} \mathrm{HCO}_{3}{ }^{-} \mathrm{m}^{-3}$ interaction caused an increment $(\mathrm{P} \leq 0.05)$ in height of young plants and reduced magnesium concentration in leaves and stems. When $30 \%$ of nitric nitrogen was replaced by ammonium nitrogen or $5 \mathrm{~mol}_{\mathrm{c}} \mathrm{HCO}_{3}^{-} \mathrm{m}^{-3}$ were added to the nutrient solution in the stage of young plant, some indicators of growth diminished but fruit yield was unaffected.

Index words: Lycopersicon esculentum, growth, nitrate/ammonium ratio, bicarbonate, hydroponics.

\section{INTRODUCCIÓN}

De los 17 nutrimentos reconocidos como esenciales para las plantas el nitrógeno ejerce el mayor efecto sobre el crecimiento (Zandstra y Liptay, 1999), y es el único nutrimento que puede ser absorbido en tres formas: aniónica $\left(\mathrm{NO}_{3}^{-}\right)$, catiónica $\left(\mathrm{NH}_{4}^{+}\right)$, molecular [CO $\left(\mathrm{NH}_{2}\right)_{2}$ ], y como aminoácidos (Näsholm et al., 2009)]. Es conocido que muchas especies de plantas incrementan su crecimiento con una combinación de nitrato y de amonio 
(Errebhi y Wilcox, 1990), comparado con fuentes individuales de $\mathrm{NO}_{3}^{-}$y $\mathrm{NH}_{4}^{+}$. En estas condiciones se incrementa la actividad de la enzima fosfoenolpiruvato carboxilasa (PEPC) en las raíces (Vuorinen et al., 1992), respuesta atribuida a una función anaplerotica de la enzima para favorecer la asimilación de amonio en las raíces mediante la producción de esqueletos carbonados que son utilizados en la síntesis de aminoácidos (Bialczyk y Lechowski, 1995), y por disminuir el transporte y acumulación de amonio en las hojas donde puede alterar muchos procesos metabólicos.

La incorporación de carbono inorgánico disuelto $\left(\mathrm{CO}_{2}\right.$ $+\mathrm{HCO}_{3}^{-}$) mediante soluciones nutritivas en la zona radical está asociada con la actividad de la PEPC (Vuorinen et al., 1992), y de esta forma influye en el metabolismo del nitrógeno al aportar esqueletos carbonados para la asimilación del amonio (Gao y Lips, 1997) o bien el bicarbonato en el citoplasma de los tejidos radicales es carboxilado en el fosfoenolpiruvato por la PEPC para sintetizar oxaloacetato, que luego es utilizado en el ciclo del ácido tricarboxílico (Cramer et al.,1996). Las plantas terrestres, además de asimilar el $\mathrm{CO}_{2}$ atmosférico en el proceso fotosintético, también pueden por las raíces absorber y asimilar el carbono inorgánico disuelto en cantidades de 1 a $5 \%$ del total de carbono asimilado por la planta (Cramer et al., 1996); la fijación de carbono inorgánico por las raíces de tomate (Lycopersicon esculentum Mill.), puede ser una fuente de carbono para la asimilación de amonio (Cramer y Lips, 1995).

En sistemas hidropónicos que utilizan agregados como sustitutos del suelo, como por ejemplo la grava (materiales con diámetro de partícula mayor de $3 \mathrm{~mm}$ ), la concentración de carbono inorgánico disuelto puede ser muy baja, lo que puede afectar el crecimiento de raíces de plantas con capacidad de incorporar al $\mathrm{HCO}_{3}{ }^{-}$en ácidos orgánicos (Cramer y Lips, 1995). En plántulas de tomate la adición de $\mathrm{HCO}_{3}^{-}$a soluciones nutritivas ha incrementado la absorción de nitratos al suministrar esqueletos carbonados para los iones amonio generados en la reducción de nitratos (Gao y Lips, 1997). Algunos factores cuyos efectos sobre el crecimiento de las plantas no han sido suficientemente investigados son la aplicación combinada de amonio y carbono inorgánico disuelto (Siddiqi et al., 2002), la concentración de carbono inorgánico disuelto (Bialczyk et al., 1996) y la forma de nitrógeno utilizada (Cramer y Richards, 1999). Los objetivos de este estudio fueron determinar el efecto de tres relaciones nitrato/amonio y tres concentraciones de bicarbonato, en la solución nutritiva, en el crecimiento de plantas y el rendimiento de tomate crecido en hidroponía.

\section{MATERIALES Y MÉTODOS}

\section{Experimento 1}

Semillas de tomate cv. 'Slolly F-1' de crecimiento indeterminado, tipo bola, fueron sembradas en charolas de poliestireno de 200 cavidades con volumen individual de $30 \mathrm{~cm}^{3}$; como sustrato se utilizó una mezcla de turba y vermiculita $(1: 1 \mathrm{v} / \mathrm{v})$. El estudio se hizo en invernadero con una duración de $35 \mathrm{~d}$ y temperaturas promedio de $38 / 25^{\circ} \mathrm{C}$ (día/noche). Cinco días después de la siembra (emergencia de plántulas) se inició la aplicación de las soluciones nutritivas (SN), cuyas concentraciones se incrementaron cada $10 \mathrm{~d}$ en el orden: 50, 75 y $100 \%$. Los tratamientos evaluados consistieron en nueve SN, resultantes de la combinación de dos factores: (1) relación porcentual nitrato/amonio (100/0, 85/15 y 70/30), y (2) concentración de $\mathrm{HCO}_{3}^{-}\left(0,2.5\right.$ y $\left.5 \mathrm{~mol}_{\mathrm{c}} \mathrm{m}^{-3}\right)$ (Cuadro 1).

En la preparación de tratamientos se utilizó como base el tratamiento uno, correspondiente a la solución universal de Steiner. Es decir, los tratamientos con 15 y 30 $\%$ del total de nitrógeno en forma de amonio tuvieron 12 $\mathrm{mol}_{\mathrm{c}} \mathrm{NO}_{3}{ }^{-} \mathrm{m}^{-3}$ x $0.15=1.8 \mathrm{~mol}_{\mathrm{c}} \mathrm{NH}_{4}{ }^{+} \mathrm{m}^{-3}$ y $3.6 \mathrm{~mol}_{\mathrm{c}} \mathrm{NH}_{4}{ }^{+}$ $\mathrm{m}^{-3}$, respectivamente. Posteriormente, se hicieron los ajustes para que los tratamientos tuvieran una presión osmótica de 0.72 atm; por tanto, las concentraciones propuestas de bicarbonato tuvieron las concentraciones finales de 0, 2.4 y $4.7 \mathrm{~mol}_{\mathrm{c}} \mathrm{HCO}_{3}{ }^{-} \mathrm{m}^{-3}$ (Cuadro 1).

Las soluciones nutritivas se prepararon con sales inorgánicas grado reactivo y agua destilada y se les añadieron las concentraciones de micronutrimentos (en $\mathrm{mg} \mathrm{L}^{-1}$ ) siguientes: Fe 2.5, Mn 0.5, B 0.5, Cu 0.02 y $\mathrm{Zn}$ 0.05. El Fe se proporcionó como Fe-EDTA y el pH de las soluciones nutritivas se ajustó a $5.5 \pm 0.1$ con $\mathrm{HCl} 1 \mathrm{~N}$ o $\mathrm{NaOH} 1 \mathrm{~N}$. Los tratamientos se distribuyeron en un diseño experimental completamente al azar con cuatro repeticiones por tratamiento, con un arreglo de tratamientos factorial $3^{2}$, para un total de 36 unidades experimentales, donde cada unidad experimental consistió de 30 plantas desarrolladas en las charolas de poliestireno. Las variables evaluadas fueron: número de 
hojas completamente formadas; altura de planta, medida desde la base del tallo hasta el ápice, con un escalímetro; diámetro de tallo, medido inmediatamente debajo de los cotiledones con un vernier digital; volumen de raíz, por desplazamiento de volumen de agua en una probeta graduada; peso fresco de hojas, tallos y raíces, cuantificados con una balanza analítica después de extraer las plantas de la charola y quitarle el sustrato adherido a las raíces con agua potable; y peso seco de hojas, tallos y raíces, después de haber permanecido estos órganos en una estufa con circulación forzada de aire a una temperatura de $70{ }^{\circ} \mathrm{C}$ durante $48 \mathrm{~h}$. Se seleccionaron 16 plántulas por tratamiento, y se integraron en cuatro repeticiones compuestas, cada una con cuatro plántulas, las que se fraccionaron en hojas y tallos. A estos órganos se les realizó el análisis químico para determinar las concentraciones de $\mathrm{N}, \mathrm{P}, \mathrm{K}, \mathrm{Ca}$ y $\mathrm{Mg}$ conforme a las metodologías reportadas por Motsara y Roy (2008). El análisis de varianza de las variables de respuesta se realizó para los factores principales y su interacción, con el programa SAS Institute (1999) versión 8.

\section{Experimento 2}

Se hizo en condiciones de casa sombra con malla de $50 \%$ (porcentaje de luz que bloquea la malla). Se utilizaron plantas de tomate de $46 \mathrm{~d}$ después de la siembra, provenientes del Experimento 1, previamente sometidas a los tratamientos descritos en el Cuadro 1. Las plantas se trasplantaron en un sistema hidropónico de subirrigación en circuito cerrado, integrado por dos recipientes de plástico con capacidad de $20 \mathrm{~L}$ cada uno, pintados de color negro en el exterior e interconectados entre sí con manguera de plástico de $1.25 \mathrm{~cm}$ de diámetro. Uno de los recipientes contenía $18 \mathrm{~L}$ de tezontle negro como sustrato con una granulometría de 3.36 a $25.4 \mathrm{~mm}$ de diámetro; la caracterización física del tezontle utilizado se indica en el Cuadro 2.

En un recipiente se colocó una planta de tomate, conducida a un tallo, y en el otro $10 \mathrm{~L}$ de solución nutritiva del tratamiento correspondiente (Cuadro 1) para efectuar dos riegos diarios al tezontle, el primero a las 8 am y el segundo a las $13 \mathrm{pm}$; diariamente se repuso el agua evapotranspirada, mediante aforo con agua destilada; el $\mathrm{pH}$ de las soluciones nutritivas se ajustó a $5.5 \pm 0.1$ con $\mathrm{HCl}$ o $\mathrm{NaOH} 1 \mathrm{~N}$; las soluciones nutritivas se renovaron cada $15 \mathrm{~d}$ y se prepararon como se describió en el Experimento 1. Los tratamientos se distribuyeron en un diseño experimental completamente al azar con un arreglo de tratamientos factorial $3^{2} \mathrm{y}$ cinco repeticiones. El periodo de cosecha duró $38 \mathrm{~d}$, e inició el 23 de enero y terminó el 2 de marzo de 2009. Las variables evaluadas fueron: rendimiento ( $\mathrm{kg} / \mathrm{planta}$ ) de frutos maduros y contenido de nutrimentos en hojas, tallos y frutos. En el último corte de frutos las plantas se cosecharon y separaron en hojas, tallos y frutos a los cuales se les determinaron las concentraciones de $\mathrm{N}, \mathrm{P}, \mathrm{K}, \mathrm{Ca}$ y $\mathrm{Mg}$, con los métodos descritos en el Experimento 1. El análisis de varianza de las variables de respuesta se hizo para los factores principales y su interacción, con el programa SAS Institute (1999) versión 8.

\section{RESULTADOS Y DISCUSIÓN}

\section{Experimento 1}

\section{Variables de crecimiento}

La interacción de los factores $\mathrm{NO}_{3}^{-} / \mathrm{NH}_{4}^{+}$x $\mathrm{HCO}_{3}{ }^{-}$ afectó en forma significativa la altura de plantas (Cuadro 3). La mayor altura, $23.5 \mathrm{~cm}$ (Figura 1), se obtuvo con la combinación nitrato/amonio 70/30 y $0 \mathrm{~mol}_{\mathrm{c}} \mathrm{HCO}_{3}{ }^{-} \mathrm{m}^{-3}$, la cual superó $(\mathrm{P} \leq 0.05)$ a las combinaciones $85 / 15$ y 0 , $70 / 30$ y $2.5,100 / 0$ y $5,85 / 15$ y 0 mol $_{\mathrm{c}} \mathrm{HCO}_{3}^{-} \mathrm{m}^{-3}$, con 20.3 , 20.2, 19.5 y $20.1 \mathrm{~cm}$, respectivamente. La mayor elongación del tallo, obtenida con la relación 70/30, aunada a los menores valores de peso seco de raíz (PSR) y volumen radical (VR), incrementa la susceptibilidad de estas plantas al acame o caída por acción del viento y del agua (Cuadro 1). La relación $\mathrm{NO}_{3}{ }^{-} / \mathrm{NH}_{4}{ }^{+}$afectó estadísticamente $(\mathrm{P} \leq 0.05)$ al PSR, al VR, y a la relación vástago/raíz (V/R). Con la relación 70/30, PSR y VR fueron $0.12 \mathrm{~g}$ y $0.23 \mathrm{~mL}$, valores menores en comparación con el PSR de $0.14 \mathrm{~g}$ obtenido con $85 / 15$ y el VR de 0.26 $\mathrm{mL}$ obtenido con 100/0. 
Cuadro 1. Composición química de las soluciones nutritivas utilizadas en los experimentos.

\begin{tabular}{ccccccccc}
\hline Tratamiento & $\mathrm{NO}_{3}^{-}$ & $\mathrm{H}_{2} \mathrm{PO}_{4}^{-}$ & $\mathrm{SO}_{4}^{2-}$ & $\mathrm{HCO}_{3}^{-}$ & $\mathrm{K}^{+}$ & $\mathrm{Ca}^{2+}$ & $\mathrm{Mg}^{2+}$ & $\mathrm{NH}_{4}^{+}$ \\
& -0 & 1.0 & 7.0 & 0 & 7.0 & 9.0 & 4.0 & 0 \\
\hline 1 & 12.0 & 1.2 & 8.6 & 0 & 6.4 & 8.2 & 3.7 & 1.8 \\
2 & 10.3 & 1.5 & 10.3 & 0 & 5.8 & 7.5 & 3.3 & 3.6 \\
3 & 8.5 & 0.7 & 4.6 & 2.4 & 6.7 & 8.7 & 3.9 & 0 \\
4 & 11.6 & 0.9 & 6.2 & 2.4 & 6.2 & 8.0 & 3.5 & 1.8 \\
5 & 9.9 & 1.1 & 7.8 & 2.4 & 5.6 & 7.2 & 3.2 & 3.6 \\
6 & 8.2 & 0.4 & 2.5 & 4.7 & 6.5 & 8.4 & 3.7 & 0 \\
7 & 11.2 & 0.6 & 4.0 & 4.7 & 6.0 & 7.7 & 3.4 & 1.8 \\
8 & 9.6 & 0.8 & 5.5 & 4.7 & 5.4 & 7.0 & 3.1 & 3.6 \\
9 & 7.9 & 0.6 & & & & \\
\hline
\end{tabular}

Cuadro 2. Caracterización físicat del tezontle utilizado en el Experimento 2.

\begin{tabular}{ccccc}
\hline $\begin{array}{c}\text { Densidad aparente } \\
\left(\mathrm{g} \mathrm{cm}^{-3}\right)\end{array}$ & $\begin{array}{c}\text { Densidad real } \\
\left(\mathrm{g} \mathrm{cm}^{-3}\right)\end{array}$ & $\begin{array}{c}\text { Porosidad total } \\
(\% \text { de volumen })\end{array}$ & $\begin{array}{c}\text { Porosidad de aireación } \\
(\% \text { de volumen })\end{array}$ & $\begin{array}{c}\text { Capacidad de retención } \\
\text { de agua (\% de volumen })\end{array}$ \\
\hline 0.66 & 2.1 & 68.9 & 54.1 & 14.8
\end{tabular}

${ }^{\mathrm{t}}$ Valor promedio de cuatro determinaciones.

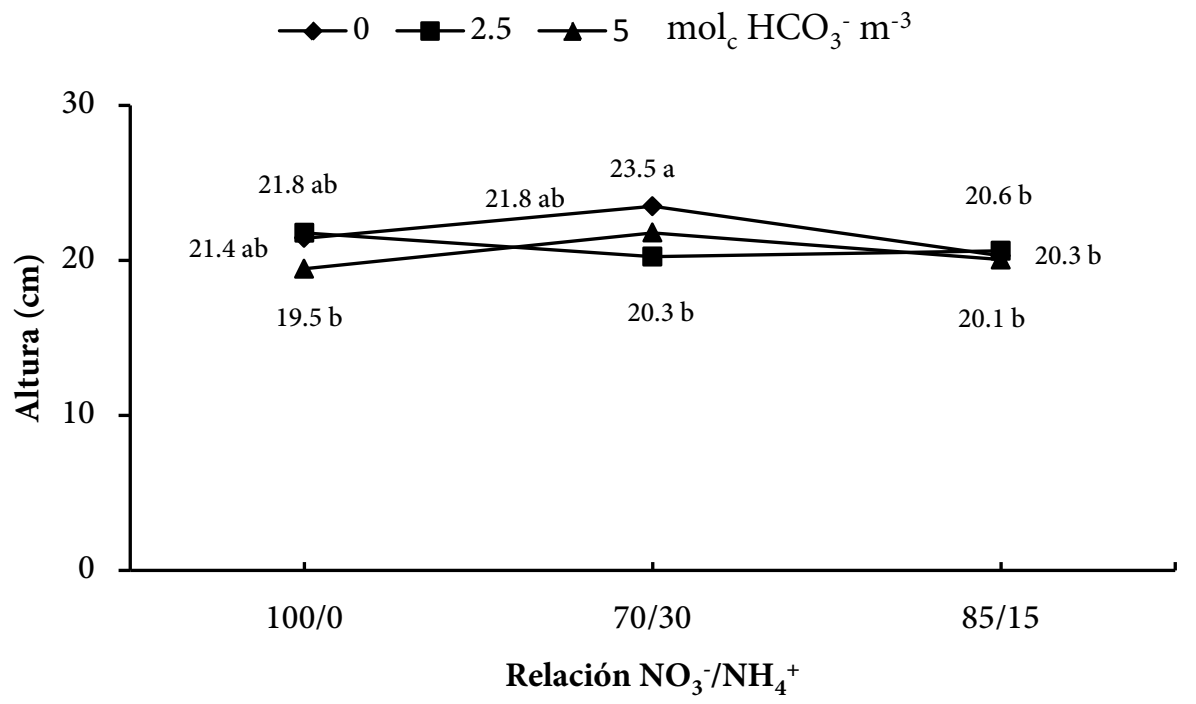

Figura 1. Efecto de la interacción relación nitrato/amonio y concentración de bicarbonato en la solución nutritiva sobre la altura de plantas jóvenes de tomate. Puntos con letras iguales en cada columna y en cada línea no son estadísticamente diferentes (Tukey, 0.05)

Sandoval et al. (1993) reportaron que al aplicar mezclas de amonio y nitrato en trigo (Triticum aestirum) en etapas tempranas de desarrollo, los pesos secos de raíz y de hojas disminuyeron notablemente. Según Marschner
(1995), el crecimiento radical puede afectarse por una alta concentración amoniacal, lo cual coincide con los menores valores de PSR y VR encontrados en este estudio. Bialczyk et al. (2004b) reportaron que el crecimiento de 
plantas de tomate es afectado cuando el amonio es la fuente única de nitrógeno, atribuible al efecto tóxico del amonio en plantas jóvenes de tomate. En este estudio los resultados sugieren que las raíces de plantas de tomate también pueden tener alta sensibilidad a la relación nitrato/amonio, ya que con la relación 70/30 se redujo el PSR y el VR. Con la relación 70/30 se obtuvo el mayor valor de la relación $\mathrm{V} / \mathrm{R}$, el cual fue 7.3; es decir, el peso seco del vástago fue 7.3 veces superior al PSR. La superioridad obtenida con 70/30 con respecto a $85 / 15$ se debe a que con la relación 70/30 disminuyó $(\mathrm{P} \leq 0.05)$ el peso seco de raíz, mientras que los pesos secos de hojas y de tallos fueron estadísticamente iguales a los obtenidos con la relación 85/15.

La concentración de $5 \mathrm{~mol}_{\mathrm{c}} \mathrm{m}^{-3}$ de $\mathrm{HCO}_{3}^{-}$en la solución nutritiva disminuyó $(\mathrm{P} \leq 0.05)$ al diámetro de tallo (DT), al peso seco de hojas (PSH), al VR, y a la V/R, comparado con $0 \mathrm{~mol}_{\mathrm{c}} \mathrm{m}^{-3}$ de $\mathrm{HCO}_{3}$ (Cuadro 3). El diámetro de tallo es un indicador del vigor de las plantas porque refleja la acumulación de fotosintatos, los cuales posteriormente pueden traslocarse a los sitios de demanda (Preciado et al., 2002). Con $5 \mathrm{~mol}_{\mathrm{c}} \mathrm{m}^{-3}{\mathrm{de} \mathrm{HCO}_{3}}^{-}$el PSH y el peso seco de planta (PSP) fueron disminuidos en 14.55 $\%$ y $9.4 \%$, respectivamente, comparados con $0 \mathrm{~mol}_{\mathrm{c}} \mathrm{m}^{-3}$ de $\mathrm{HCO}_{3}{ }^{-}$en la solución nutritiva.

Barhoumi et al. (2007) reportaron una reducción en el PSP de dos cultivares de chícharo (Pisum sativum L.) por efecto de $10 \mathrm{~mol}_{c} \mathrm{~m}^{-3}$ de $\mathrm{HCO}_{3}{ }^{-}$, atribuido a una deficiencia de hierro inducida por incrementos de $\mathrm{pH}$ ocasionados por los aportes de nitrógeno en forma de nitratos. En el presente estudio el $\mathrm{pH}$ de las soluciones nutritivas diariamente se ajustó a $5.5 \pm 0.1$ por lo que se descarta alcalinización de las soluciones que pudiera generar una deficiencia de hierro. Se puede inferir entonces que la concentración de $5 \operatorname{mol}_{\mathrm{c}} \mathrm{m}^{-3}$ de bicarbonato ocasionó un antagonismo con el fósforo, lo que limitó su absorción porque los contenidos de este elemento resultaron reducidos $(\mathrm{P} \leq 0.05)$ en hojas y en tallos, lo cual posiblemente ocasionó menor peso seco de hojas y menor diámetro de tallo (Cuadro 4). Pissaloux et al., (1995) reportaron que el bicarbonato redujo la absorción de fósforo en plantas de lupino (Lupinus polyphyllus L.). Además de lo anterior, es conocido que las plantas con bajos contenidos de fósforo tienen menor crecimiento del vástago y la relación de peso seco entre el vástago/raíz es generalmente baja (Mengel y Kirkby,
2000), lo que coincide con lo encontrado en este estudio ya que la menor relación $(\mathrm{V} / \mathrm{R}=6.1)$ se obtuvo con $5 \mathrm{~mol}_{\mathrm{c}}$ $\mathrm{m}^{-3}$ de bicarbonato (Cuadro 3 ).

\section{Concentración de nutrimentos en órganos vegetales}

La interacción de los factores $\mathrm{NO}_{3}^{-} / \mathrm{NH}_{4}^{+} \times \mathrm{HCO}_{3}{ }^{-}$ afectó $(\mathrm{P} \leq 0.05)$ la concentración de $\mathrm{Mg}$ en hojas y en tallos (Cuadro 4). Con 0 mol $_{c} \mathrm{HCO}_{3}^{-} \mathrm{m}^{-3}$ y una relación nitrato/amonio de 70/30 la concentración de magnesio en hojas fue $0.59 \%$, valor menor que el obtenido con 0 mol $_{c}$ $\mathrm{HCO}_{3}{ }^{-} \mathrm{m}^{-3}$ y que con las relaciones 100/0 y 85/15 (1.07 y $1.05 \%$, respectivamente) (Figura 2). Esto puede atribuirse a que la inclusión de amonio en ciertas concentraciones puede limitar la absorción de otros cationes, como el magnesio, y su contenido en la planta (Lasa et al., 2000; Mengel y Kirkby, 2000). Es por ello que la menor concentración de $\mathrm{Mg}$ se obtuvo con la relación 70/30; en contraste, la mayor (1.07 \%) se obtuvo con la relación $100 / 0$, ya que en estas condiciones el nitrato es el principal anión absorbido y debe predominar en la savia del xilema, por lo que requiere de cantidades equivalentes de cationes, entre los cuales está el magnesio para balancear la carga negativa del nitrato (Mengel y Kirkby, 2000).

$\mathrm{Al}$ incrementar de 0 a $5 \operatorname{mol}_{\mathrm{c}} \mathrm{HCO}_{3}{ }^{-} \mathrm{m}^{-3}$ en la solución nutritiva, la concentración de magnesio en las hojas con la relación 70/30 aumentó $49 \%$, pues de $0.59 \%$ subió a 0.88 \% (Figura 2), valor estadisticamente igual a los obtenidos con las relaciones $85 / 15$ y $100 / 0$ (0.74 y $0.63 \%)$. En la Figura 3 se muestra que con la relación $85 / 15$ y 5 mol $_{c}$ $\mathrm{HCO}_{3}{ }^{-} \mathrm{m}^{-3}$ el contenido de $\mathrm{Mg}$ en tallos fue de $1.39 \%$, valor mayor $(\mathrm{P} \leq 0.05)$ que el obtenido con esta misma relación y $0 \mathrm{~mol}_{\mathrm{c}} \mathrm{HCO}_{3}{ }^{-} \mathrm{m}^{-3}(0.84 \%)$. Tal aumento en la concentración de $\mathrm{Mg}$ en hojas y tallos sugiere que hay un sinergismo del bicarbonato con el magnesio, tal vez por un incremento en la asimilación del nitrógeno amoniacal en aminoácidos, en donde el bicarbonato aporta, vía la PEPc, esqueletos carbonados adicionales que reducen la toxicidad del amonio (Roosta y Schjoerring, 2008), catión inhibidor de la absorción de magnesio.

La relación $\mathrm{NO}_{3}{ }^{-} / \mathrm{NH}_{4}{ }^{+}$afectó la concentración de calcio en hojas (Cuadro 4). Con la relación 70/30 la concentración de Ca fue de $1.01 \%$, valor menor $(\mathrm{P} \leq 0.05)$ que con 100/0 y 85/15 (1.72 y $1.37 \%$ ). Lo anterior puede explicarse al considerar que a mayor concentración de amonio en la solución nutritiva, hay mayor absorción de 
este ion, el cual es un catión que es absorbido rápidamente; en cambio, la absorción de calcio (Lasa et al., 2000) y magnesio es más lenta, lo que significa que las plantas extraen cationes y aniones del medio nutrititivo en cantidades desiguales, lo que afecta sus contenidos en las plantas (Mengel y Kirkby, 2000). Siddiqi et al. (2002) reportaron que la presencia de 50\% del nitrógeno total de la solución nutritiva en forma de amonio redujo significativamente las concentraciones de calcio $y$ magnesio en hojas, lo que coincide con lo encontrado en este estudio.

Cuadro 3. Efecto de la relación porcentual de $\mathrm{NO}_{3}{ }^{-} / \mathrm{NH}_{4}{ }^{+}$y concentraciones de $\mathrm{HCO}_{3}^{-}$en la solución nutritiva sobre altura (A), diámetro de tallo (DT), número de hojas (NH), peso seco de hojas (PSH), peso seco de tallos (PST), peso seco de raíz (PSR), peso seco de planta (PSP), volumen de raíz (VR), y relación vástago/raíz (V/R) de plantas jóvenes de tomate.

\begin{tabular}{|c|c|c|c|c|c|c|c|c|c|}
\hline Factor & $\begin{array}{l}\text { A } \\
(\mathrm{cm})\end{array}$ & $\begin{array}{l}\text { DT } \\
(\mathrm{mm})\end{array}$ & $\mathrm{NH}$ & $\begin{array}{l}\text { PSH } \\
(\mathrm{g})\end{array}$ & $\begin{array}{l}\text { PST } \\
(\mathrm{g})\end{array}$ & $\begin{array}{l}\text { PSR } \\
(\mathrm{g})\end{array}$ & $\begin{array}{l}\text { PSP } \\
(\mathrm{g})\end{array}$ & $\begin{array}{l}\text { VR } \\
(\mathrm{mL})\end{array}$ & $\mathrm{V} / \mathrm{R}$ \\
\hline & \multicolumn{9}{|c|}{ Relación porcentual de $\mathrm{NO}_{3}{ }^{-} / \mathrm{NH}_{4}^{+}$} \\
\hline $85 / 15$ & $20.3 \mathrm{~b}$ & $3.1 \mathrm{a}$ & $6.5 \mathrm{a}$ & $0.51 \mathrm{a}$ & $0.36 \mathrm{a}$ & $0.14 \mathrm{a}$ & $1.03 \mathrm{a}$ & $0.24 \mathrm{ab}$ & $6.2 \mathrm{~b}$ \\
\hline $70 / 30$ & $21.8 \mathrm{a}$ & $3.1 \mathrm{a}$ & $6.4 \mathrm{a}$ & $0.51 \mathrm{a}$ & $0.38 \mathrm{a}$ & $0.12 \mathrm{~b}$ & $1.01 \mathrm{a}$ & $0.23 \mathrm{~b}$ & $7.3 \mathrm{a}$ \\
\hline 2.5 & $20.9 \mathrm{ab}$ & $3.1 \mathrm{ab}$ & $6.4 \mathrm{a}$ & $0.52 \mathrm{ab}$ & $0.38 \mathrm{a}$ & $0.13 \mathrm{a}$ & $1.02 \mathrm{a}$ & $0.26 \mathrm{a}$ & $7.1 \mathrm{a}$ \\
\hline 5.0 & $20.4 \mathrm{~b}$ & $2.9 \mathrm{~b}$ & $6.1 \mathrm{a}$ & $0.47 \mathrm{~b}$ & $0.34 \mathrm{a}$ & $0.13 \mathrm{a}$ & $0.97 \mathrm{a}$ & $0.21 \mathrm{~b}$ & $6.1 \mathrm{~b}$ \\
\hline & \multicolumn{9}{|c|}{$\mathrm{NO}_{3}^{-} / \mathrm{NH}_{4}^{+} \times \mathrm{HCO}_{3}^{-}$} \\
\hline & $* *$ & ns & ns & ns & ns & ns & ns & ns & ns \\
\hline
\end{tabular}

${ }^{\mathbf{t}}$ Medias con letras iguales no son estadísticamente diferentes (Tukey, 0.05$) ; \mathrm{ns}=$ no significativo; ${ }^{* *}=\mathrm{P} \leq 0.01$.

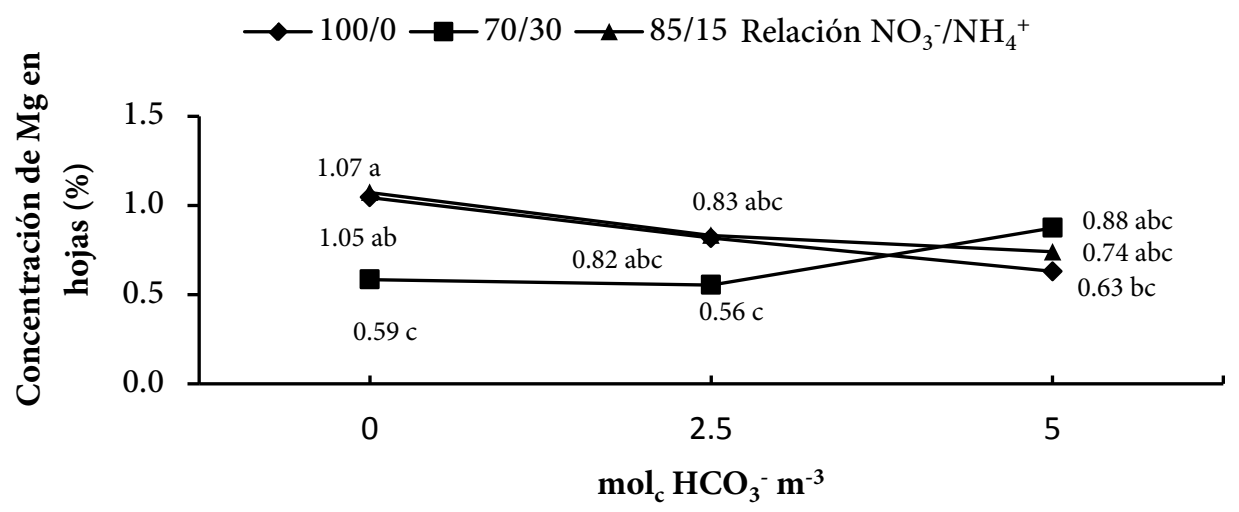

Figura 2. Efecto de la interacción relación nitrato/amonio y concentración de bicarbonato en la solución nutritiva sobre el contenido de magnesio en hojas de plantas jóvenes de tomate. Puntos con la letras iguales en cada columna y en cada línea no son estadísticamente diferentes (Tukey, 0.05). 


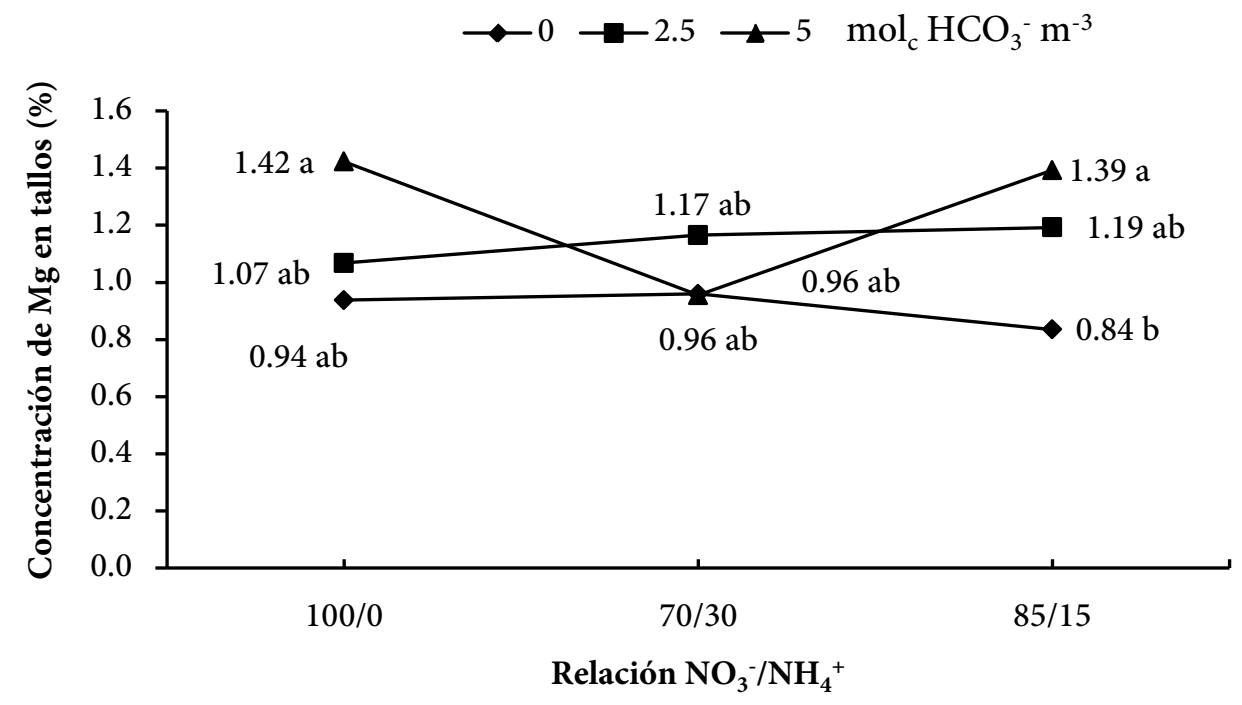

Figura 3. Efecto de la interacción relación nitrato/amonio y concentración de bicarbonato en la solución nutritiva sobre el contenido de magnesio en tallos de plantas jóvenes de tomate. Puntos con la letras iguales en cada columna y en cada línea no son estadísticamente diferentes (Tukey, 0.05).

Cuadro 4. Efecto de la relación $\mathrm{NO}_{3}{ }^{-} / \mathrm{NH}_{4}{ }^{+}$y concentraciones de $\mathrm{HCO}_{3}{ }^{-}$en la solución nutritiva sobre la concentración de nutrimentos en los órganos de las plantas de tomate (Experimento 1 ).

\begin{tabular}{|c|c|c|c|c|c|}
\hline \multirow[t]{3}{*}{ Factor } & $\mathrm{N}$ & $\mathrm{P}$ & $\mathrm{K}$ & $\mathrm{Ca}$ & \multirow[t]{2}{*}{$\mathrm{Mg}$} \\
\hline & \multirow{2}{*}{\multicolumn{5}{|c|}{ Hojas }} \\
\hline & & & & & \\
\hline & \multicolumn{5}{|c|}{ Relación porcentual de $\mathrm{NO}_{3}{ }^{-} / \mathrm{NH}_{4}^{+}$} \\
\hline $100 / 0$ & $5.01 \mathrm{a}^{9}$ & $0.10 \mathrm{a}$ & $3.85 \mathrm{a}$ & $1.72 \mathrm{a}$ & $0.83 \mathrm{ab}$ \\
\hline $85 / 15$ & $5.01 \mathrm{a}$ & $0.16 \mathrm{a}$ & $3.77 \mathrm{a}$ & $1.37 \mathrm{~b}$ & $0.88 \mathrm{a}$ \\
\hline \multirow[t]{2}{*}{$70 / 30$} & $5.00 \mathrm{a}$ & $0.17 \mathrm{a}$ & $3.72 \mathrm{a}$ & $1.01 \mathrm{c}$ & $0.67 \mathrm{~b}$ \\
\hline & \multicolumn{5}{|c|}{$\mathrm{HCO}_{3}^{-}\left(\mathrm{mol}_{\mathrm{c}} \mathrm{m}^{-3}\right)$} \\
\hline 0 & $5.05 \mathrm{a}$ & $0.25 \mathrm{a}$ & $3.65 \mathrm{a}$ & $1.01 \mathrm{c}$ & $0.90 \mathrm{a}$ \\
\hline 2.5 & $4.90 \mathrm{a}$ & $0.15 \mathrm{~b}$ & $3.75 \mathrm{a}$ & $1.42 \mathrm{~b}$ & $0.74 \mathrm{a}$ \\
\hline \multirow[t]{5}{*}{5.0} & $5.07 \mathrm{a}$ & $0.10 \mathrm{c}$ & $3.93 \mathrm{a}$ & $1.62 \mathrm{a}$ & $0.75 \mathrm{a}$ \\
\hline & \multicolumn{5}{|c|}{$\mathrm{NO}_{3}^{-} / \mathrm{NH}_{4}^{+} \times \mathrm{HCO}_{3}^{-}$} \\
\hline & ns & ns & ns & ns & ** \\
\hline & \multicolumn{5}{|c|}{ Tallos } \\
\hline & \multicolumn{5}{|c|}{ Relación porcentual de $\mathrm{NO}_{3}^{-} / \mathrm{NH}_{4}^{+}$} \\
\hline 0 & $2.86 \mathrm{~b}$ & $0.16 \mathrm{~b}$ & $3.39 \mathrm{a}$ & $0.91 \mathrm{a}$ & $1.14 \mathrm{a}$ \\
\hline $85 / 15$ & $3.10 \mathrm{a}$ & $0.22 \mathrm{ab}$ & $3.46 \mathrm{a}$ & $0.74 \mathrm{~b}$ & $1.14 \mathrm{a}$ \\
\hline \multirow[t]{2}{*}{$70 / 30$} & $2.90 \mathrm{ab}$ & $0.26 \mathrm{a}$ & $3.25 \mathrm{a}$ & $0.62 \mathrm{~b}$ & $1.02 \mathrm{a}$ \\
\hline & \multicolumn{5}{|c|}{$\mathrm{HCO}_{3}^{-}\left(\mathrm{mol}_{\mathrm{c}} \mathrm{m}^{-3}\right)$} \\
\hline 0 & $3.07 \mathrm{a}$ & $0.29 \mathrm{a}$ & $3.45 \mathrm{a}$ & $0.60 \mathrm{c}$ & $0.91 \mathrm{~b}$ \\
\hline 2.5 & $3.09 \mathrm{a}$ & $0.22 \mathrm{~b}$ & $3.46 \mathrm{a}$ & $0.75 \mathrm{~b}$ & $1.15 \mathrm{a}$ \\
\hline \multirow[t]{3}{*}{5.0} & $2.70 \mathrm{~b}$ & $0.13 c$ & $3.27 \mathrm{a}$ & $0.92 \mathrm{a}$ & $1.26 \mathrm{a}$ \\
\hline & \multicolumn{5}{|c|}{$\mathrm{NO}_{3}^{-} / \mathrm{NH}_{4}^{+} \times \mathrm{HCO}_{3}^{-}$} \\
\hline & ns & ns & ns & ns & $* *$ \\
\hline
\end{tabular}

${ }^{9}$ Medias con letras iguales no son estadísticamente diferentes (Tukey, 0.05 ); ns = no significativo; ${ }^{* *}=\mathrm{P} \leq 0.01$. 
La concentración de $\mathrm{HCO}_{3}{ }^{-}$en la solución nutritiva afectó $(\mathrm{P} \leq 0.05)$ las concentraciones de fósforo y calcio en hojas y las concentraciones de nitrógeno, fósforo y calcio en tallos (Cuadro 4). Con $0 \mathrm{~mol}_{\mathrm{c}} \mathrm{HCO}_{3}^{-} \mathrm{m}^{-3}$ las concentraciones de $\mathrm{P}$ en hojas y tallos fueron de $0.25 \mathrm{y}$ $0.29 \%$, valores superiores $(\mathrm{P} \leq 0.05)$ a los obtenidos con 2.5 y $5.0 \mathrm{~mol}_{\mathrm{c}} \mathrm{HCO}_{3}^{-} \mathrm{m}^{-3}, 0.15$ y $0.10 \% \mathrm{P}$ en hojas, respectivamente, y 0.22 y $0.13 \% \mathrm{P}$ en tallos. Esto sugiere un posible antagonismo entre el bicarbonato y el fósforo, como fue explicado en las variables del crecimiento, ya que conforme aumentan las concentraciones de bicarbonato en la solución nutritiva las concentraciones de fósforo en hojas y tallos disminuyen. Con $0 \mathrm{~mol}_{c}$ $\mathrm{HCO}_{3}^{-} \mathrm{m}^{-3}$ las concentraciones de $\mathrm{Ca}$ en las hojas y en tallos fueron de $1.01 \%$ y $0.60 \%$, valores menores $(\mathrm{P} \leq$ $0.05)$ a los obtenidos con 2.5 y 5.0 mol $_{\mathrm{c}} \mathrm{HCO}_{3}^{-} \mathrm{m}^{-3}$, de 1.42 y $1.62 \%$ de Ca en hojas, y de 0.75 y $0.92 \%$ de Ca en tallos, lo que sugiere un posible sinergismo entre el bicarbonato y el calcio, como fue explicado anteriormente para el caso de magnesio. Es decir, que al aumentar la concentración de bicarbonato en la solución nutritiva se incrementan también las concentraciones de calcio en hojas y tallos.

La relación $\mathrm{NO}_{3}{ }^{-} / \mathrm{NH}_{4}{ }^{+}$afectó $(\mathrm{P} \leq 0.05)$ las concentraciones de nitrógeno y de fósforo en los tallos. Con 85/15 se obtuvo una concentración de nitrógeno de $3.1 \%$, valor estadísticamente igual al obtenido con $70 / 30$ (2.9 \%) pero mayor $(\mathrm{P} \leq 0.05)$ que con $100 / 0(2.86 \%)$. Con 70/30 la concentración de fósforo fue $0.26 \%$, valor mayor comparado con la de $0.16 \%$ obtenido con 100/0. Este resultado coincide con lo reportado por Lasa et al., (2000), quienes encontraron que plantas de girasol (Helianthus annuus L.) suministradas con amonio presentaron mayores contenidos de fósforo que las plantas abastecidas con nitrato; también coincide con Marschner (1995), quien menciona que las plantas suministradas con nitrógeno amoniacal a menudo contienen altas concentraciones de aniones, como fósforo, para mantener el equilibrio de aniones y cationes en las plantas. La concentración de $\mathrm{HCO}_{3}^{-}$en la solución nutritiva afectó $(\mathrm{P} \leq 0.05)$ la concentración de nitrógeno en tallos. Con $5.0 \mathrm{~mol}_{\mathrm{c}} \mathrm{HCO}_{3}{ }^{-} \mathrm{m}^{-3}$ la concentración fue de $2.7 \%$, menor a la registrada con $0 \mathrm{~mol}_{\mathrm{c}} \mathrm{HCO}_{3}{ }^{-} \mathrm{m}^{-3}$, que fue de 3.07 \%. Según Bialczyk et al. (1994), la absorción de nitrógeno puede estar limitada por la concentración de bicarbonatos en el medio nutritivo, y una mayor concentración de nitrógeno puede ser obtenida con el nivel más adecuado de $\mathrm{HCO}_{3}^{-}$, que para esta variable fue 0 y $2.5 \mathrm{~mol}_{\mathrm{c}} \mathrm{HCO}_{3}{ }^{-} \mathrm{m}^{-3}$.

\section{Experimento 2}

\section{Rendimiento de tomate}

En esta variable no hubo efecto significativo de los factores relación nitrato/ amonio y concentración de bicarbonato en la solución nutritiva (Cuadro 5). Con respecto al efecto de la relación nitrato/amonio, estos resultados difieren de lo reportado por Bialczyk et al. (2007) y Siddiqi et al. (2002), quienes observaron que al añadir 10 y $20 \%$ del nitrógeno total en forma de $\mathrm{NH}_{4}{ }^{+}$a la solución nutritiva, el rendimiento de tomate se incrementó en 15 y $20 \%$, comparado con el tratamiento de $100 \%$ de nitrógeno nítrico; esto puede atribuirse a que los cultivares de tomate responden de forma diferente a las fuentes de nitrógeno (Ben-Oliel et al., 2005), o diferencias en las condiciones ambientales (Kotsiras et al., 2005) prevalecientes en estos estudios, y a las concentraciones de amonio utilizadas.

Con relación al efecto de la concentración de bicarbonato en la solución nutritiva, este resultado coincide con lo reportado por Siddiqi et al. (2002); pero difiere de lo reportado por Bialczyk et al. (2007), quienes mencionan que las diferencias encontradas en los efectos del bicarbonato en el rendimiento pueden estar asociadas con las condiciones experimentales, como $\mathrm{pH}$, composición del medio y otras condiciones ambientales. En este estudio y en el de Siddiqi et al. (2002), los pH de las soluciones nutritivas se ajustaron a $5.5 \pm 0.1$ y 5.8 , respectivamente, mientras que Bialczyk et al. (2007) utilizaron un $\mathrm{pH}$ de 6.9. Estas variaciones en el $\mathrm{pH}$ afectan la ionización de las diferentes formas de carbonato y sus concentraciones en el equilibrio químico del sistema $\mathrm{CO}_{2}-\mathrm{H}_{2} \mathrm{O}$ (Lindsay, 1977).

De igual manera, la respiración de las raíces y de los microorganismos produce $\mathrm{CO}_{2}$ que baja el $\mathrm{pH}$ y modifica la solubilidad de las formas de carbonato. Además, la porosidad del sustrato puede alterar el intercambio de $\mathrm{CO}_{2}$ entre el sustrato y la atmósfera, y afectar el equilibrio de los carbonatos. Los resultados de este estudio muestran la posibilidad de reducir el uso de fertilizantes nitrogenados en la solución nutritiva en forma de nitratos 
al sustituir $30 \%$ de ellos por amonio, sin afectar el rendimiento del tomate $\mathrm{cv}$. 'Slolly F-1' en casa sombra, lo que representaría un ahorro en los costos de producción, porque los fertilizantes amoniacales son más baratos que los nítricos.

\section{Concentración de nutrimentos en órganos vegetales}

La relación nitrato/amonio afectó $(\mathrm{P} \leq 0.05)$ la concentración de calcio en hojas y de calcio y magnesio en tallos (Cuadro 6). Con la relación 70/30 la concentración de calcio en hoja fue $2.51 \%$ y en tallos fue $0.55 \%$, valores menores $(\mathrm{P} \leq 0.05)$ que los obtenidos con 100/0 (3.86 y $1.03 \%)$; en cambio, la concentración de magnesio en tallos con la relación $70 / 30$ fue $0.29 \%$, valor menor ( $\mathrm{P} \leq$ $0.05)$ que el obtenido con 100/0 (0.45\%). Estos resultados coinciden con los obtenidos en el Experimento 1 y con lo reportado por Siddiqi et al. (2002), y podría explicarse por la forma y nivel de nutrición nitrogenada que pueden influir en el equilibrio de aniones y cationes en las plantas. Es decir, al aumentar los niveles de amonio se estimula su absorción y la de los aniones que compensan su carga. En contraste, las plantas suministradas con amonio tienen concentraciones más bajas de cationes inorgánicos, como calcio, magnesio y potasio (Ten Hoopen et al., 2010; Mengel y Kirkby, 2000).

Con la relación 70/30 la concentración de fósforo en tallos fue $0.23 \%$, valor mayor $(\mathrm{P} \leq 0.05)$ al obtenido con 100/0 (0.10 \%), y coincide con lo encontrado en el Experimento 1. La concentración de bicarbonato en la solución nutritiva afectó $(\mathrm{P} \leq 0.05)$ las concentraciones de nitrógeno, calcio y potasio en hojas, y de fósforo, calcio y magnesio en tallos (Cuadro 6). Con $5 \mathrm{~mol}_{\mathrm{c}} \mathrm{HCO}_{3}{ }^{-} \mathrm{m}^{-3}$ las concentraciones de nitrógeno y calcio se incrementaron en 46.9 y $85.1 \%$, respectivamente, en comparación con 0 $\mathrm{mol}_{\mathrm{c}} \mathrm{HCO}_{3}^{-} \mathrm{m}^{-3}$; por el contrario, la concentración de potasio se redujo en $24.1 \%$. Bialczyk et al. (2004a) mencionaron que la asimilación del carbono inorgánico en las células radicales puede afectar el metabolismo del nitrógeno, por lo que un aumento en dicha asimilación en las raíces puede estar asociado con el incremento de compuestos nitrogenados incorporados. El aumento de calcio en hojas coincide con los resultados del Experimento 1, lo cual indicaría un efecto similar de la concentración de $5 \mathrm{~mol}_{\mathrm{c}} \mathrm{HCO}_{3}^{-} \mathrm{m}^{-3}$ en ambos experimentos.
Con $0 \mathrm{~mol}_{\mathrm{c}} \mathrm{HCO}_{3}{ }^{-} \mathrm{m}^{-3}$ la concentración de potasio en las hojas fue $2.53 \%$, valor mayor $(\mathrm{P} \leq 0.05)$ al obtenido con $5 \mathrm{~mol}_{\mathrm{c}} \mathrm{HCO}_{3}^{-} \mathrm{m}^{-3}$ (1.92\%). Un efecto similar fue reportado en chícharo (Barhoumi et al. 2007). En contraste, Bialczyk et al. (1994) reportaron que al aplicar $5.68 \mathrm{~mol}_{\mathrm{c}} \mathrm{HCO}_{3}{ }^{-} \mathrm{m}^{-3}$ a plantas de tomate la concentración de potasio en hojas y peciolo se incrementó significativamente, atribuible a los mayores contenidos de este elemento presentes en floema y xilema.

Con $5 \mathrm{~mol}_{\mathrm{c}} \mathrm{HCO}_{3}{ }^{-} \mathrm{m}^{-3}$ la concentración de fósforo en tallos fue de $0.08 \%$, valor menor $(\mathrm{P} \leq 0.05)$ que los obtenidos con 0 y 2.5 mol $_{c} \mathrm{HCO}_{3}^{-} \mathrm{m}^{-3}$ (0.27 y $\left.0.14 \%\right)$. Estos resultados coinciden con lo encontrado en el Experimento 1, lo que sugiere un posible antagonismo entre bicarbonato y fósforo. La relación nitrato/amonio afectó $(\mathrm{P} \leq 0.05)$ la concentración de fósforo en los frutos; con la relación 70/30 este valor fue $0.25 \%$, valor mayor al obtenido con la relación 100/0 (0.19\%), lo cual podrá deberse a que el amonio estimula la absorción de fósforo (Mengel y Kirkby, 2000). Con $5 \mathrm{~mol}_{\mathrm{c}} \mathrm{HCO}_{3}^{-} \mathrm{m}^{-3}$ la concentración de fósforo fue $0.20 \%$, menos que el obtenido con $0 \mathrm{~mol}_{\mathrm{c}} \mathrm{HCO}_{3}^{-} \mathrm{m}^{-3}(0.24 \%)$.

Cuadro 5. Efecto de la relación nitrato/amonio y la concentración de bicarbonatos en la solución nutritiva en el rendimiento acumulado de tomate (38 d de cosecha).

\begin{tabular}{cc}
\hline Factor & $\begin{array}{c}\text { Rendimiento } \\
(\mathrm{kg} / \text { planta })\end{array}$ \\
\hline Relación nitrato/amonio & \\
$100 / 0$ & $4.347 \mathrm{a}$ \\
$85 / 15$ & $4.503 \mathrm{a}$ \\
$70 / 30$ & $4.147 \mathrm{a}$ \\
$\mathrm{HCO}_{3}^{-}\left(\mathrm{mol}_{\mathrm{c}} \mathrm{m}^{-3}\right)$ & \\
0 & $4.369 \mathrm{a}$ \\
2.5 & $4.252 \mathrm{a}$ \\
5 & $4.376 \mathrm{a}$ \\
nitrato/amonio $\mathrm{x} \mathrm{HCO}{ }^{-}$ & $\mathrm{ns}$ \\
\hline Medias con letras iguales dentro de cada columna no son \\
estadísticamente diferentes, Tukey $(\mathrm{P} \leq 0.05) ; \mathrm{ns}=$ no significativo.
\end{tabular}


Cuadro 6. Efecto de la relación nitrato/amonio y la concentración de bicarbonatos en la solución nutritiva sobre la concentración de nitrógeno, fósforo, potasio, calcio y magnesio en órganos de tomate (Experimento 2).

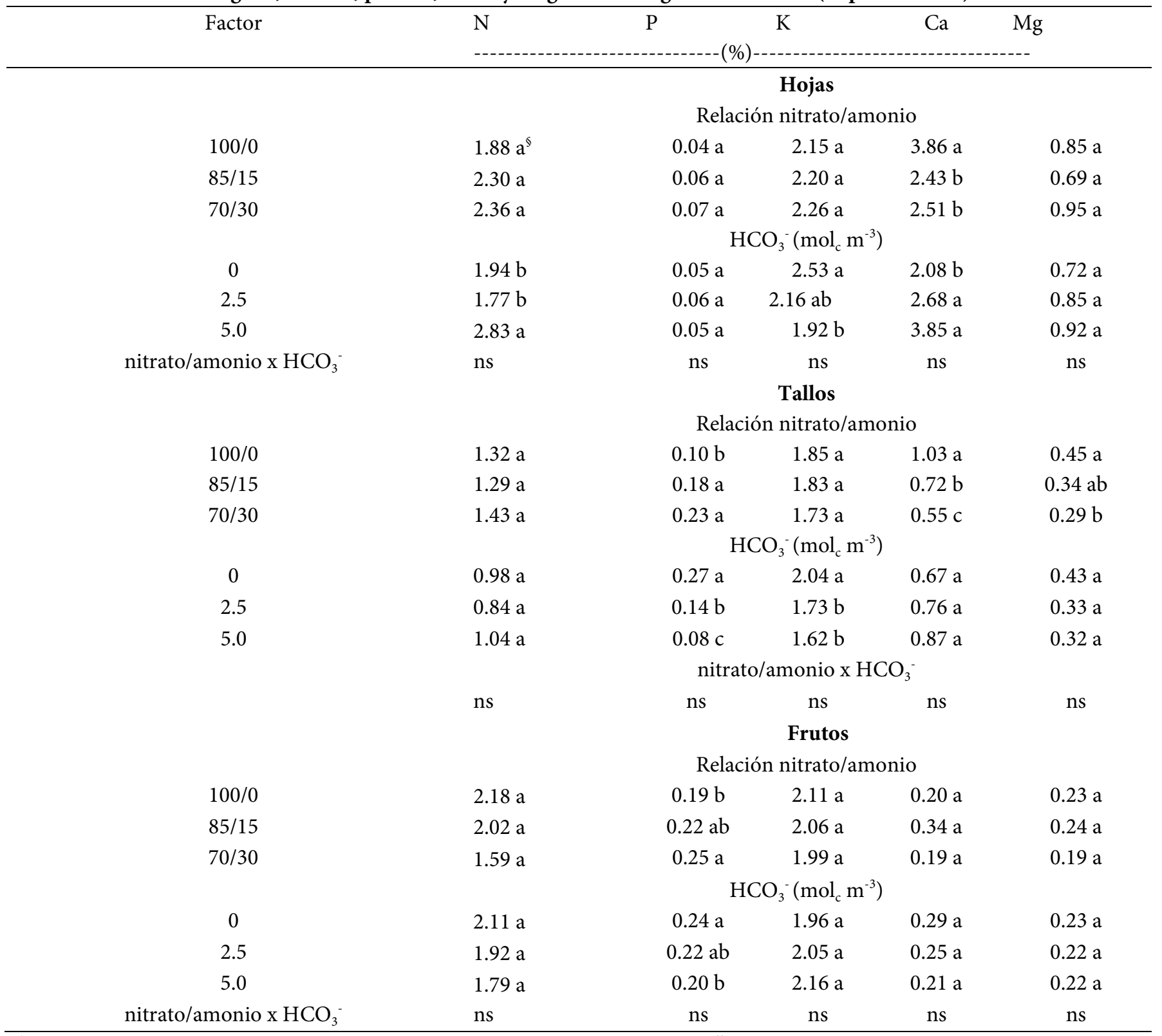

${ }^{5}$ Medias con letras iguales no son estadísticamente diferentes (Tukey, 0.05 ); ns $=$ no significativo; ${ }^{* *}=\mathrm{P} \leq 0.01$.

\section{CONCLUSIONES}

En plantas jóvenes de tomate la relación porcentual $70 / 30$ de nitrato/amonio en la solución nutritiva disminuyó el peso seco de raíz y el volumen radical, mientras que la concentración de $5 \mathrm{~mol}_{\mathrm{c}} \mathrm{HCO}_{3}^{-} \mathrm{m}^{-3}$ en la solución nutritiva redujo el diámetro de tallo, el peso seco de hojas, el volumen radical y la relación vástago/raíz. La relación nitrato/amonio afectó en forma inversamente proporcional la concentración de calcio en hojas y tallos de plantas jóvenes y plantas adultas. En contraste, dicha relación afectó en forma directamente proporcional la concentración de fósforo en hojas y tallos de plantas jóvenes y plantas adultas. La concentración de $\mathrm{HCO}_{3}{ }^{-}$en la solución nutritiva afectó en forma directamente proporcional la concentración de calcio, pero inversamente proporcional a la concentración de fósforo en hojas y tallos de plantas jóvenes y plantas adultas. 
Tanto las plantas jóvenes como las plantas adultas del tomate cv. 'Slolly F-1' presentaron efectos similares en las concentraciones de calcio y de fósforo en hojas y tallos por acción de la menor relación nitrato/amonio, o por la mayor concentración de bicarbonato. La sustitución de 30 $\%$ del total de nitrógeno nítrico con un porcentaje similar de nitrógeno amoniacal en la solución nutritiva, redujo algunos indicadores del crecimiento de tomate pero no al rendimiento, lo que permitiría el uso de amonio antes y después del trasplante de tomate en casa sombra, y reducir así los costos de producción.

\section{AGRADECIMIENTOS}

A la Universidad Autónoma de Sinaloa por su apoyo financiero, a través del Programa de Fortalecimiento y Apoyo a Proyectos de Investigación (PROFAPI-2009).

\section{BIBLIOGRAFÍA}

Barhoumi Z, M Rabhi, M Gharsalli (2007) Effects of two nitrogen forms on the growth and iron nutrition of pea cultivated in presence of bicarbonate. J. Plant Nutr. 30:1953-1965.

Ben-Oliel G, S Kant, M Naim, H D Rabinowitch, G R Takeoka, G Buttery, U Kafkafi (2005) Effects of ammonium to nitrate ratio and salinity on yield and fruit quality of large and small tomato fruit hybrids. J. Plant Nutr. 27:1795-1812.

Bialczyk J, Z Lechowski, A Libik (1994) Growth of tomato seedlings under different $\mathrm{HCO}_{3}$ concentration in the medium. J. Plant Nutrition 17:801-816.

Bialczyk J, Z Lechowski (1995) Chemical composition of xylem sap of tomato grown on bicarbonato containing médium. J. Plant Nutr. 18:2005-2021.

Bialczyk J, Z Lechowski, A Libik (1996) Fruiting of tomato cultivated on medium enriched with bicarbonate. J. Plant Nutr. 19:305321.

Bialczyk J, Z Lechowski, D Dziga (2004a) Composition of the xylem sap of tomato seedlings cultivated on media with $\mathrm{HCO}_{3}{ }^{-}$and $\mathrm{N}$ source as nitrate or ammonium. Plant Soil 263:265-272.

Bialczyk J, Z Lechowski, A Libik (2004b) Early vegetative growth of tomato plants in media containing nitrogen source as nitrate, ammonium, or various nitrate-ammonium mixtures with bicarbonate addition. J. Plant Nutr. 27:1687-1700

Bialczyk J, Z Lechowski, D Dziga, E Mej (2007) Fruit yield of tomato cultivated on media with bicarbonate and nitrate/ammonium as the nitrogen source. J. Plant Nutr. 30:149-161.

Cramer M D, S H Lips (1995) Enriched rhizosphere $\mathrm{CO}_{2}$ concentrations can ameliorate the influence of salinity on hydroponically grown tomato plants. Physiol. Plant. 94:425-432.

Cramer M D, N Savidov, S H Lips (1996) The influence of enriched rhizosphere $\mathrm{CO}_{2}$ concentrations on $\mathrm{N}$ uptake and metabolism in NR-deficient and wild type barley. Physiol. Plant. 97:47-54.
Cramer M D, M B Richards (1999) The effect of rhizosphere disolved inorganic carbon on gas exchange characteristics and growth rates of tomato seedlings. J. Exp. Bot. 50:79-87.

Errebhi M, G E Wilcox (1990) Plant species response to ammoniumnitrate concentration rations. J. Plant Nutr. 13:1017-1029.

Gao Z F, S H Lips (1997) Effects of increasing inorganic carbon supply to roots on net nitrate uptake and assimilation in tomato seedlings. Physiol. Plant. 101:206-212.

Kotsiras A, C M- Olympios, H C Passam (2005) Effects of nitrogen form and concentration on yield and quality of cucumbers grown on rockwool during spring and winter in southern Greece. J. Plant Nutr. 28:2027-2035.

Lasa B, S Frechilla, M Aleu, B G Moro, C Lamsfus, P M AparicioTrejo (2000) Effects of low and high levels of magnesium on the response of sunflower plants grown with ammonium and nitrate. Plant Soil 225:167-174.

Lindsay W L (1979) Chemical Equilibria in Soils. John Wiley \& Sons, Inc. USA. pp:78-84.

Marschner H (1995) Mineral Nutrition of Higher Plants. 2nd ed. Academic Press. San Diego, California, USA. pp:229-255.

Mengel K, E A Kirkby (2000) Principios de Nutrición Vegetal. Trad. al español de RJ Melgar y M Ruíz. 4a ed. (1987) Internacional Potash Institute. Basel, Switzerland. pp:113-145.

Motsara M R, R N Roy (2008) Guide to Laboratory Establishment for Plant Nutrient Analysis. FAO Fertilizer and Plant Nutrition Bulletin 19. Food an Agriculture Organization of the United Nations. pp:220.

Näsholm T, K Kielland, U Ganeteg (2009) Uptake of organic nitrogen by plant. New Phytol. 182:31-48.

Pissaloux A, P Morard, G Bertoni (1995) Alkalinity-bicarbonatecalcium effects on iron chlorosis in white lupine in soilless culture. In: J. Abadia (ed). Iron Nutrition in Soils and Plants. Springer-Verlag, New York, USA. pp:127-133.

Preciado R P, G A B Castillo, J L T Torres, J K Shibata, L T Chávez, A M Garza (2002) Nitrógeno y potasio en la producción de plántulas de melón. Terra 20:67-76.

Roosta H R, J K Schjoerring (2008) Root carbon enrichment alleviates ammonium toxicity in cucumber plants. J. Plant Nutr. 31:941958.

Sandoval V M, G Alcántar G, J L Tirado (1993) Momento oportuno de la aplicación de amonio y nitrato para incrementar la producción de grano de trigo. Terra 11:61-66.

SAS Institute (1999) Statistical Analysis System (SAS) Versión 8. Cary, N. C. USA.

Siddiki M Y, B Malhotra, X Min, A D M Glass (2002) Effects of ammonium and inorganic carbon enrichment on growth and yield of a hydroponic tomato crop. J. Plant Nutr. Soil Sci. 165:191-197.

Ten Hoopen F, T A Cuin, P Pedas, J N Hegelund, S Shabala, J K Schjoerring, T P Jahn (2010) Competition between uptake of ammonium and potassium in barley and Arabidopsis roots: molecular mechanisms and physiological consequences. J. Exp. Bot. 61:2303-2315.

Vuorinen A H, E M Vapaavuori, R O Lapinjoki (1992) Metabolism of inorganic carbon taken up by roots in salix plants. J. Exp. Bot. 43:789-795.

Zandstra J W, A Liptay (1999) Nutritional effects on transplant root and shoot growth- A review. Acta Hort. 504:23-32. 